Research Article

\title{
Low Cost Optical Sensing Device for Fuel Detection in Ships
}

\author{
P. Papadopoulou ${ }^{1}$, L. Magafas ${ }^{1}$, P. Agelopoulos ${ }^{1}$, N. $\operatorname{Vordos}^{1}$ and K. Vlachos ${ }^{2}$. \\ ${ }^{1}$ Electrical Engineering Department, Eastern Macedonia and Thrace Institute of Technology, St. Lucas, 65404, Kavala, Greece. \\ ${ }^{2}$ Computer Engineering and Informatics Department, University of Patras, GR26500, Rio, Patra, Greece.
}

Received 23 November 2015; Accepted 13 May 2016

\begin{abstract}
In the present work the development of a new, very low cost, simple to manufacture and use, optical sensing device for remote, on line detection of the type of fuels used in ships, is presented. The main goal of this optical sensing device is the on line detection of the fuel optical absorption that is used by the ship. The basic operating principle of the proposed sensor is based on different absorption in the range of visible spectrum between bunker diesel and fuel oil. Experimental measurements, using monochromatic laser light or white led light, have shown that the proposed sensor can distinguish very accurately the difference between the two types of oil, giving the advantage to detect the type of fuel.
\end{abstract}

Keywords: Optical Sensing Devices; Ship Fuel Detection

\section{Introduction}

Ships can use two different types of fuels: diesel or fuel oil. In particular, ships are using diesel close to coasts and fuel Oil when they are away. Considering that the Diesel is higher cost than fuel oil, but less polluting, based on their maritime regulations near coast should be used diesel and the sea, and away from inhabited areas, fuel oil, shipping companies would like to know in real time the type of fuel that their ships use according to distance from ports or residential areas [1], [2].

According to literature [4] properties that can be used in order to detect the type of fuel that ships use might be focused on the following are:

a) Fuel's density, diesel density varies from $0.890 \mathrm{gr} / \mathrm{cm}^{3}$ to $0.930 \mathrm{gr} / \mathrm{cm}^{3}$, on the other hand fuel oils density varies from $0.960 \mathrm{gr} / \mathrm{cm}^{3}$ to $1.010 \mathrm{gr} / \mathrm{cm}^{3}$.

b) Fuel's Viscosity, the values for diesel are from 1.5 cst to $6.0 \mathrm{cst}$, whereas for fuel Oil are from $180 \mathrm{cst}$ to $380 \mathrm{cst}$.

c) Fuel's Index of Refraction, where 1.445 is for diesel and 1.554 for fuel oil.

d) The optical absorption in the visible spectrum.

e) The fluorescence spectrum (Different tops Diesel - Diesel).

The most common on-line measuring systems are systems that can measure, density, refractive index, viscosity, aromatic components etc. Also spectrophotometers are often used.

The recent progress and development has thrived a whole on-line technology measurement of various properties of fuels based on their spectroscopic data. For example, the determination of the aromatic constituents of the fuel is accomplished by on-line spectrophotometer FTIR (Fourier

*E-mail address: ppapado@teikav.edu.gr ISSN: 1791-2377@ 2016 Eastern Macedonia and Thrace Institute of Technology. All rights reserved.
Transform Infra-Red), and the determination of various other properties by NIR analyzers and other chemical optical sensor devices due to their efficiency [4]. Today, there are also plenty of integrated systems for on line fuel detection in ships based in technologies presented above, however their cost varies from $10000 €$ up to $26000 €$ [2].

The aim of this work is to show the feasibility of a cost efficient simple to manufacture and use, optical sensing device for remote, on line detection of the type and quality of fuels used in ships. The operating principle of the optical sensing device based on direct sensing scheme, where some intrinsic optical property such as absorption, in our case, of samplesis monitored for sensing purposes. The interesting part of the present work is the continuous and real time monitoring of the type of fuels used in ships. Especially, if the proposed optical sensing device used in conjunction with the density sensing device proposed in previous work [5], the type, the quality and the amount of sulfur (S) of the fuels used in ships will be detected more accurately.

\section{The Optical Sensing Device}

An important technique which gives basic information about materials properties in liquid, gaseous and solid state is the technique of absorption spectroscopy, where specialized spectrophotometers used in order to estimate the absorption of the investigated material. The absorption spectroscopy obeys the Lambert-Beer law:

$I=I_{0} e^{-a c}$

Where:

$A=a c=\log \frac{I}{I_{0}}$

- $\mathrm{A}$ is the absorbance of the sample

- $\mathrm{I}_{0}$ is the incident light intensity 
- I is the intensity of the propagated light

- $\quad \ell$ is the sample thickness

- $\quad \mathrm{c}$ is the concentration of the sample

- $\alpha$ is the absorption coefficient of the sample

Using the technique of the absorption spectroscopy the absorption spectrum of diesel and fuel oil were measured. More specific, a ship diesel sample and a fuel oil sample were measured, using a UV-VIS absorption spectrophotometer, in the range from $250 \mathrm{~nm}$ to $850 \mathrm{~nm}$. Figure 1 shows the absorption spectrums of ship diesel and fuel oil samples.

It is obvious from this figure that fuel oil exhibits a much higher absorption compared to ship diesel over the range of the visible spectrum $(400 \mathrm{~nm}-800 \mathrm{~nm})$. This high absorption is due to the composition of fuel oil, which is a complex mixture of high molecular weight hydrocarbons, and also compounds of sulfur, nitrogen and oxygen. The components of the fuel oil absorbing more in the visible spectrum than the ship diesel, which is a mixture of aliphatic, cycloaliphatic and aromatic hydrocarbons $\mathrm{C} 12$ - C25 carbon atoms [6].

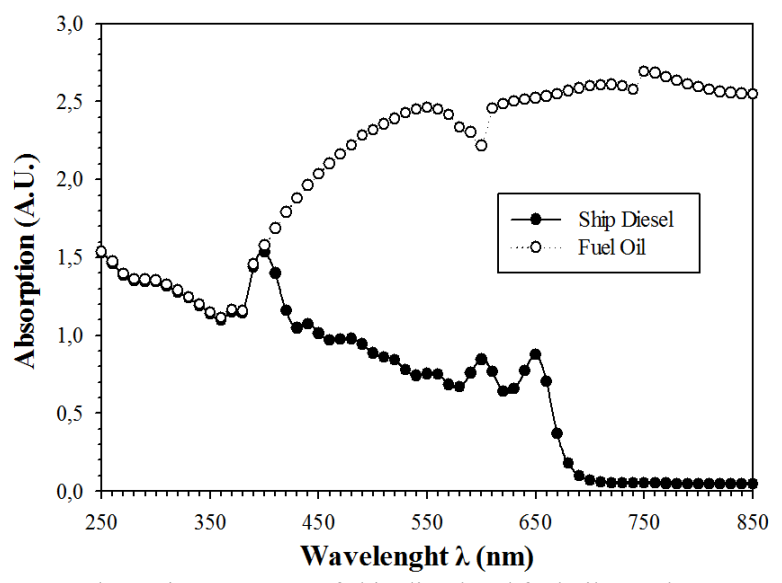

Fig. 1. Absorption spectrum of ship diesel and fuel oil samples

The proposed optical sensing device based in the different absorption that ship diesel and fuel oil exhibits in the range of visible spectrum and design taking into account low cost, simple to manufacture and use implementation. The absorption - based optical sensing device consist of,

- A light source that it could be a diode laser or a LED as low cost light source with wavelength in the range of red thus about $630 \mathrm{~nm}$.

- A sample cell, which is a glass cylindrical tube

- Optical detector, which is a photo-resistance

This optical sensing device could be connected with a"collecting" microcontroller, and the measurement data are transmitted wirelessly to Laros Networks, which is a new innovative, reliable and low cost platform for data acquisition and evaluation.

Figure 2 shows, a block diagram of the optical sensing device implementation. The sensing device placed parallel to the central fuel supply pipe, the fuel absorbs part of the incident light, the propagated light incidence the optical detector. The measurement rom the detector is driven to microcontroller and compared with measurements of the direct light source. The ship diesel gives low photo resistance measurements due to low absorption in the corresponding wavelength, while the fuel oil gives high photo resistance measurements similar to the dark light conditions due to high absorption. With a proper calibration of the optical sensing system, optical device and microcontroller, the type of fuels can be estimated accurately. Laros wireless network provides online complete identification of fuels type and transit early warnings of the ship's status to the administration headquarters.

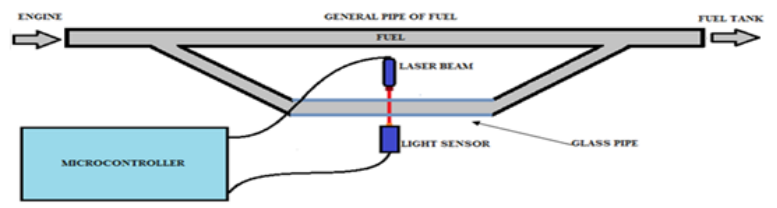

Figure 2. Block diagram of the proposed optical sensing device.

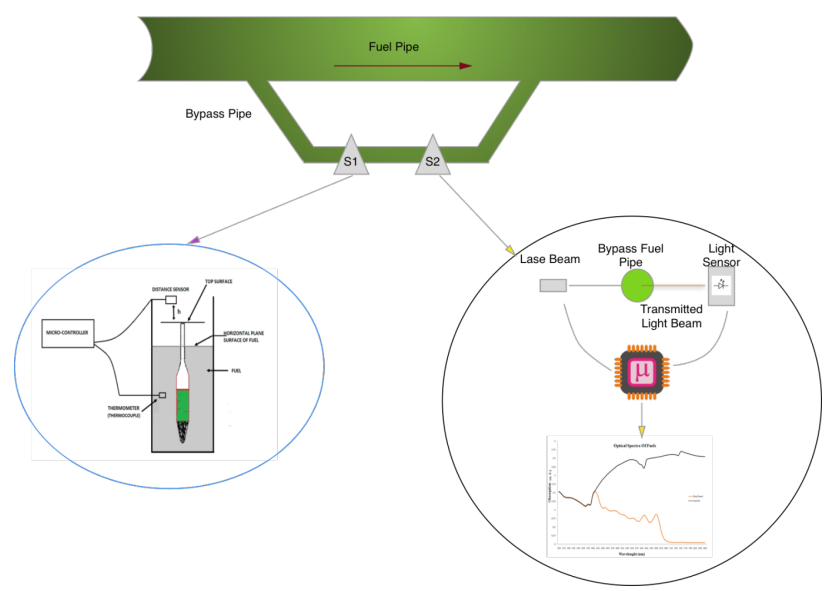

Figure 3. Block diagram of the combined optical and density sensing device.

Since the whole optical sensing device constructed by low cost materials and components, the total coast was limited by the wireless Laros network connection. In any case the total coast of the proposed optical sensing device is lower one or two order of magnitude than that of other systems available in the international market and can be estimated up to $200 \$$.

In conjunction with the density sensing device proposed in previous work [5] the accuracy of fuel type detection becomes stricter eliminating potential error due to double cross checked measurements, further more the Sulfur concentration in the fuel can be estimated. In the case where the optical and density sensing devices are combined the total cost of these structure is also very low up to $500 \$$. Figure 3 shows a block diagram of the combined optical and density sensing devices.

\section{Conclusions}

In the present work, a low cost optical sensing device system was designed and developed. The proposed system is able to detect on-line the type of the fuel used in a ship, using measurements based in fuels optical absorption. This sensing device was constructed based on the different optical absorption that ship diesel and fuel oil exhibit. The optical sensing device is convenient with Laros platform suitable for on line transition of ship's fuel status, in real time, to administration headquarters. In the case that this optical sensing device could be combined with the density sensing device [5], can give the type of the fuel as well as and the possible Sulfur concentration in the fuel. 


\section{Acknowledgments}

Authors acknowledge financial support to the research project "Ship's Health Condition, Operation Status and Performance Remote Monitoring Based on wireless sensor network and technical experience management system - MariBrain" in the frame of Synergasia 2011, Greek Ministry of Culture, Education and Religious Affairs, General Secretariat For Research \& Technology.

\section{References}

1. Paula J. Spreutels and Monique B. Vermeire Ghent, "Everything you need to know about marine fuels", in Fuel an Matinetime Marketing, Belgium, UK, 2001.

2. Klaus Kuhlgatz, Mochael F. Benzel,"System and Method for Total Management of Ships" Patent US20080147257 A1, 2006.

3. James, G. Speight, "The Chemistry and Technology of Petroleum", Taylor and Francis Group, LLG, 2014.

4. HummadHabibQazi, Abu Bakar bin Mohammad, and Muhammad Akram, "Recent Progress in Optical Chemical Sensors", Sensors, 12, pp. 16522-16556, 2012.

5. L. Magafas, "Low Cost Sensing Device for Fuel Detection in Ships", MOCOST 2015.

6. D.A.Skoog,F.J.Holler,T.A.Nieman,"Principles ofInstrumental Anal ysis",SaundersCollegePublishing,5th Edition, New York, 1997. 